\title{
Correlation Energy of an Electron Gas at High Density*
}

\author{
Murray Gelt-Mann, Department of Physics, California Institute of Technology, Pasadena, California \\ AND \\ Keith A. Brueckner, Department of Physics, University of Pennsylvania, Philadelphia, Pennsylvania
}

(Received December 14, 1956)

\begin{abstract}
The quantity $\epsilon_{c}$ is defined as the correlation energy per particle of an electron gas expressed in rydbergs. It is a function of the conventional dimensionless parameter $r_{s}$, where $r_{s}{ }^{-3}$ is proportional to the electron density. Here $\epsilon_{c}$ is computed for small values of $r_{s}$ (high density) and found to be given by $\epsilon_{c}=A \ln r_{s}$ $+C+O\left(r_{s}\right)$. The value of $A$ is found to be 0.0622 , a result that could be deduced from previous work of Wigner, Macke, and Pines. An exact formula for the constant $C$ is given here for the first time; earlier workers had made only approximate calculations of $C$. Further, it is shown how the next correction in $r_{\mathrm{s}}$ can be computed. The method is based on summing the most highly divergent terms of the perturbation series under the integral sign to give a convergent result. The summation is performed by a technique similar to Feynman's methods in field theory.
\end{abstract}

$\mathrm{W}^{\mathrm{E}}$ consider the idealized problem of the groundstate energy of a gas of electrons in the presence of a uniform background of positive charge that makes the system neutral. For most practical problems, of course, the uniform positive charge must be replaced by a lattice of positive ions, but we shall not treat this more realistic case.

We have, then, a fully degenerate Fermi-Dirac system with Coulomb interactions. Let us employ the conventional notation for the problem. The inverse density or volume per electron is set equal to $\frac{4}{3} \pi r_{0}{ }^{3}$. The dimensionless parameter $r_{s}$ is defined as $r_{0}$ divided by the Bohr radius. The ground state energy per particle in rydbergs is called $\epsilon$ and is a function of $r_{s}$ only, since there are no other dimensionless quantities involved.

We shall compute $\epsilon$ in the case of high density or small $r_{s}$. Since $r_{s}$ is proportional to $e^{2}$, an expansion in powers of $r_{s}$ is essentially an expansion in powers of $e^{2}$, that is, the perturbation expansion. Unfortunately, a straightforward perturbation expansion leads to divergences, but let us ignore that difficulty for a moment.

The leading term in the perturbation series is evidently the Fermi energy, the kinetic energy of the degenerate free electron gas. The maximum electron momentum $P$, the radius of the Fermi sphere in momentum space, is given by $P=(9 \pi / 4)^{\frac{1}{3}} \hbar r_{0}^{-1}$. The Fermi energy per particle in rydbergs is thus

$$
\epsilon_{F}=\frac{3}{5}\left(\frac{P^{2}}{2 m}\right) / \frac{e^{4} m}{2 \hbar^{2}}=\frac{3}{5}\left(\frac{9 \pi}{4}\right)^{\frac{3}{3}} \frac{1}{r_{s}^{2}} \approx \frac{2.21}{r_{s}^{2}} .
$$

The next term in the series is the exchange energy, the expectation value of the potential energy in the ground state of a free electron gas. It is one higher order in $e^{2}$ or $r_{s}$ than the Fermi energy and so is proportional to $1 / r_{s}$. It is easily evaluated to be

$$
\epsilon_{x}=-\frac{3}{4}\left(\frac{e^{2}}{\pi}\right) P / \frac{e^{4} m}{2 \hbar^{2}}=-\frac{3}{2 \pi}\left(\frac{9 \pi}{4}\right)^{\frac{1}{3}} \frac{1}{r_{s}} \approx-\frac{0.916}{r_{s}} .
$$

* This study was performed by the authors as consultants to the RAND Corporation, Santa Monica, California, and was sponsored entirely by the U. S. Atomic Energy Commission.
If we now calculate the effect of the potential in secondorder perturbation theory, we should expect a term of one higher order in $e^{2}$ or $r_{s}$ than (2), that is, a constant independent of $r_{s}$. However, the second-order perturbation formula diverges logarithmically at small momentum transfers on account of the long-range character of the Coulomb force. Thus some refinement of perturbation theory is necessary in order to carry the computation further.

The terms in the energy beyond (1) and (2) are called collectively the "correlation energy,"

$$
\epsilon_{c} \equiv \epsilon-\epsilon_{F}-\epsilon_{x} \text {. }
$$

This name was introduced by Wigner, ${ }^{1}$ who called attention to the importance of the correlation energy in solid-state problems. Following Wigner's lead, calculations were made by $\mathrm{Macke}^{2}$ and by Pines ${ }^{3}$ that led essentially to expressions of the form

$$
\begin{aligned}
\epsilon_{c}= & \left(2 / \pi^{2}\right)(1-\ln 2) \ln r_{s}+C \\
& \quad+\text { terms that vanish as } r_{s} \rightarrow 0, \\
\approx & 0.0622 \ln r_{s}+C+\cdots,
\end{aligned}
$$

where in each case the constant $C$ was calculated approximately. ${ }^{4}$

We give here an exact evaluation of the constant $C$ by a method that should permit also the calculation of higher corrections in $r_{s}$. The basic idea of the method is to examine the increasingly divergent terms of the perturbation series and to notice that they fall into

${ }^{1}$ E. P. Wigner, Phys. Rev. 46, 1002 (1934).
2 W. Macke, Z. Naturforsch. 5a, 192 (1950).

${ }^{3}$ D. Pines, Phys. Rev. 92, 626 (1953); D. Pines, in Solid State Physics, edited by F. Seitz and D. Turnbull (Academic Press, Inc., New York, 1955), Vol. 1, p. 367.

4 Pines' result is actually not of the form of Eq. (4). However, he has neglected a term which he calls the exchange correlation energy and which adds to his result for $\epsilon$ the quantity $0.0311 \mathrm{ln} r$, $-0.0905+\epsilon_{b}^{(2)}+O\left(r_{s}\right)$, where $\epsilon_{b}^{(2)}$ is defined in our Eq. (9). When we supply this term, his final answer takes on the form of Eq. (4). When we quote Pines' result later, we mention this modification.

Macke's result does agree with Eq. (4). However, he seems to have made certain unnecessary approximations in his calculation of $C$, and we have therefore recomputed $C$, using his method, in Appendix I. 
subseries that can be summed under the integral sign to give convergent results. The logarithmic divergence in second order is then automatically replaced by a logarithmic dependence on the expansion parameter $r_{s}$, as in (4). In this respect, our method is similar to Macke's. ${ }^{2}$ However, Macke fails to sum all of the processes that contribute to the constant $C$. In our work, we are able to exhibit all of them and then to sum them by a procedure similar to Feynman's methods in field theory.

Let us discuss, then, the behavior of the formal perturbation series for $\epsilon$. The coefficient of each power of $r_{s}$ can be written as an integral over various dimensionless vectors $\mathbf{q}_{i}$, which are virtual momentum transfers divided by $P$, the Fermi momentum. The term independent of $\boldsymbol{r}_{s}$ then diverges logarithmically, as we have said. The next term, formally linear in $r_{s}$, diverges quadratically, the succeeding one quartically, etc. Now, since the correlation energy is finite, these integrals, when summed, must cut themselves off at some characteristic value of the dimensionless momentum transfers $q$. Moreover, the nature of the cutoff is clear from the results of work on the plasma vibrations of an electron gas, especially that of Bohm and Pines. ${ }^{5}$ It has been shown that collective electron motions effectively screen the Coulomb field at a distance of the order of

$$
r_{\max } \sim \text { (const.) } r_{0}^{\frac{1}{2}} a^{\frac{1}{2}}+\text { higher terms in } r_{0},
$$

where $a$ is the Bohr radius. The effective cutoff for $q$ is then

$$
q_{\min } \sim \text { (const.) } r_{s}^{\frac{1}{2}}+\text { higher terms in } r_{s} .
$$

We use this estimate in conjunction with our estimate of the correlation energy

$$
\begin{aligned}
\epsilon_{c} \sim(\log \text { divergence })+r_{s} & \text { (quadratic div. }) \\
& \left.+r_{s}^{2} \text { (quartic div. }\right)+\cdots,
\end{aligned}
$$

and we deduce the following results:

(i) $\epsilon_{c}=A \ln r_{s}+C+$ terms that vanish as $r_{s} \rightarrow 0$.

(ii) The coefficient $A$ can be found merely from the strength of the logarithmic divergence in second-order perturbation theory. This leads to the value of 0.0622 quoted above.

(iii) The only virtual processes contributing to $C$ beyond the second order are those which contribute the highest divergence in each order of perturbation theory. Those processes leading to lower divergences will give higher powers of $\boldsymbol{r}_{s}$ in the final expression for $\epsilon$.

Now the processes that lead to the highest divergences are easily identified. The divergences are caused by the piling-up of factors $1 / q^{2}$ coming from Coulomb interactions in momentum space. Evidently the greatest piling-up occurs when only a single momentum transfer is involved and this single $q$ is handed from electron to electron, contributing a factor $1 / q^{2}$ each time. Moreover, we may distinguish momentum transfers with

\footnotetext{
${ }^{5}$ D. Bohm and D. Pines, Phys. Rev. 92, 609 (1953).
}

and without exchange; when exchange occurs the factor is no longer $1 / q^{2}$ but $1 /\left(\mathbf{p}_{1}-\mathbf{p}_{2}+\mathbf{q}\right)^{2}$, where $\mathbf{p}_{1}$ and $\mathbf{p}_{2}$ are the initial electron momenta. In the case of exchange, then, the singularity at $q=0$ is not enhanced. We may therefore ignore exchange entirely beyond the second order in computing $C$.

We may now list the processes that contribute to $C$ in the first few orders of perturbation theory. In second order we must include everything; we have two terms, the logarithmically divergent one we have mentioned and a finite one coming from exchange. They may be written as follows:

$$
\begin{array}{r}
\epsilon_{a}{ }^{(2)}=-\frac{3}{8 \pi^{5}} \int \frac{d^{3} q}{q^{4}} \int_{\substack{p_{1}<1 \\
\left|\mathbf{p}_{1}+\mathbf{q}\right|>1}} d^{3} p_{1} \int_{\substack{p_{2}<1 \\
\left|\mathbf{p}_{2}+\mathbf{q}\right|>1}} d^{3} p_{2} \\
\\
\times \frac{1}{q^{2}+\mathbf{q} \cdot\left(\mathbf{p}_{1}+\mathbf{p}_{2}\right)},
\end{array}
$$

and

$$
\begin{aligned}
\epsilon_{b}(2)=\frac{3}{16 \pi^{5}} \int \frac{d^{3} q}{q^{2}} \int_{\substack{p_{1}<1 \\
\left|\mathbf{p}_{1}+\mathbf{q}\right|>1}} d^{3} p_{1} \int_{\substack{\mathbf{p}_{2}<1 \\
\left|\mathbf{p}_{2}+\mathbf{q}\right|>1}} d^{3} p_{2} \\
\\
\quad \times \frac{1}{\left(\mathbf{q}+\mathbf{p}_{1}+\mathbf{p}_{2}\right)^{2}} \frac{1}{q^{2}+\mathbf{q} \cdot\left(\mathbf{p}_{1}+\mathbf{p}_{2}\right)}
\end{aligned}
$$

In these processes two electrons in the Fermi sea with initial momenta $\mathbf{p}_{1} P$ and $-\mathbf{p}_{2} P$ have undergone a collision with momentum transfer $\mathbf{q} P$, emerging into unoccupied states with momenta $\left(\mathbf{p}_{1}+\mathbf{q}\right) P$ and $\left(-\mathbf{p}_{2}-\mathbf{q}\right) P$ and then returning to their original states. The factor $1 /\left[q^{2}+\mathbf{q} \cdot\left(\mathbf{p}_{1}+\mathbf{p}_{2}\right)\right]$ comes from the energy denominator. In (8) there is a factor $1 / q^{2}$ for each collision, while in the exchange correlation energy (9) one factor is $1 / q^{2}$ and the other $1 /\left(\mathbf{q}+\mathbf{p}_{1}+\mathbf{p}_{2}\right)^{2}$.

In writing higher order integrals, we shall not indicate explicitly the conditions $|\mathbf{p}+\mathbf{q}|>1$ and $p<1$, but these must still be obeyed by all vectors $\mathbf{p}$ to insure that initial states are occupied and others unoccupied. In third order the processes involving a single momentum transfer are these: Two electrons with momenta $\mathbf{p}_{1} P$ and $-\mathbf{p}_{2} P$ emerge from the sea into states with momenta $\left(\mathbf{p}_{1}+\mathbf{q}\right) P$ and $\left(-\mathbf{p}_{2}-\mathbf{q}\right) P$, as before. One of them now returns to its original state and transfers its excess momentum $\mathbf{q} P$ or $-\mathbf{q} P$ to a third electron, which emerges from a state with momentum $\mathbf{p}_{3} P$. This third one and the one still outstanding now return to their original states. Both third-order processes contribute equal amounts to the energy, since the first and second electrons are quite equivalent and it does not matter which one first interacts with the third electron.

The processes involved may be represented diagrammatically as in Fig. 1. In second order, two electrons called 1 and 2 are excited and then de-excited. In third order, electrons 1 and 2 are excited, one of them is de- 
excited while exciting a third electron 3 , and then the outstanding electrons are de-excited. And so forth.

The third-order contribution is given by

$$
\begin{aligned}
& \epsilon^{(3)}=2\left(\frac{\alpha r_{s}}{\pi^{2}}\right)\left(\frac{3}{8 \pi^{5}}\right) \int \frac{d^{3} q}{q^{6}} \int d^{3} p_{1} \int d^{3} p_{2} \int d^{3} p_{3} \\
& \times \frac{1}{q^{2}+\mathbf{q} \cdot\left(\mathbf{p}_{1}+\mathbf{p}_{2}\right)} \frac{1}{q^{2}+\mathbf{q} \cdot\left(\mathbf{p}_{1}+\mathbf{p}_{3}\right)},
\end{aligned}
$$

where $\alpha$ is $(4 / 9 \pi)^{\frac{1}{3}}$.

In fourth order, rather complicated processes begin to appear, as one may see from Fig. 1. In the first diagram in fourth order, electron 1 is twice replaced before de-exciting with electron 2 . In the next, electron 1 is replaced, then electron 2 is replaced, and then the de-excitation takes place. The following two diagrams are similar with electrons 1 and 2 exchanging roles. In the last two diagrams the excitation of 1 and 2 is followed by the excitation of another pair 3 and 4 ; then electrons 1 and 3 de-excite together and electrons 2 and 4 de-excite together, in either order.

The fourth-order contributions are given by

$$
\begin{gathered}
\epsilon^{(4)}=-2\left(\frac{\alpha r_{s}}{\pi^{2}}\right)^{2}\left(\frac{3}{8 \pi^{5}}\right) \int \frac{d^{3} q}{q^{8}} \int d^{3} p_{1} \int d^{3} p_{2} \int d^{3} p_{3} \int d^{3} p_{4} \\
\times\left\{\frac{1}{q^{2}+\mathbf{q} \cdot\left(\mathbf{p}_{1}+\mathbf{p}_{2}\right)} \frac{1}{q^{2}+\mathbf{q} \cdot\left(\mathbf{p}_{1}+\mathbf{p}_{3}\right)} \frac{1}{q^{2}+\mathbf{q} \cdot\left(\mathbf{p}_{1}+\mathbf{p}_{4}\right)}\right. \\
+\frac{1}{q^{2}+\mathbf{q} \cdot\left(\mathbf{p}_{1}+\mathbf{p}_{2}\right)} \frac{1}{q^{2}+\mathbf{q} \cdot\left(\mathbf{p}_{1}+\mathbf{p}_{3}\right)} \frac{1}{q^{2}+\mathbf{q} \cdot\left(\mathbf{p}_{3}+\mathbf{p}_{4}\right)} \\
+\frac{1}{q^{2}+\mathbf{q} \cdot\left(\mathbf{p}_{1}+\mathbf{p}_{2}\right)} \frac{1}{2 q^{2}+\mathbf{q} \cdot\left(\mathbf{p}_{1}+\mathbf{p}_{2}+\mathbf{p}_{3}+\mathbf{p}_{4}\right)} \\
\left.\times \frac{1}{q^{2}+\mathbf{q} \cdot\left(\mathbf{p}_{1}+\mathbf{p}_{3}\right)}\right\} .
\end{gathered}
$$

It is easy now to write down the contributions from any order. The problem then is to sum all these contributions before performing the integral over $q$. In order to see how to sum them, we note the similarity to diagrams in field theory. There, of course, pairs of electrons and holes (positrons) are under consideration, interacting with an external field. Here we consider pairs of electrons in interaction with the Fermi sea and ignore the holes. Nevertheless the similarity is sufficient for the application of Feynman's artifice of considering a pair as composed of one electron traveling forward in time and one backward in time. The creation or annihilation of a pair is interpreted as the turningaround of a particle in time. If we look back at Fig. 1 with Feynman's point of view in mind, we see that in each order all the diagrams are merely versions of one single diagram in which a single electron starts out, is replaced over and over again, and finally returns to its starting point. The various forms of the diagram come from the choice that faces the electron each time it is replaced, a choice of being replaced by an electron going forward in time or one going backward in time.

If we introduce "time variables," then, and let the electrons propagate either forward or backward in time with suitable propagators, we should be able to represent the sum of all diagrams in each order by a single integral. The integrals over the time-variables should give us the various energy denominators we need. We try as the propagator the function

$$
F_{q}(t)=\int d^{3} p \exp \left[-|t|\left(\frac{1}{2} q^{2}+\mathbf{q} \cdot \mathbf{p}\right)\right]
$$

which is arranged so that integration over positive or negative time will introduce into the energy denominator plus or minus $\left(\frac{1}{2} q^{2}+\mathbf{q} \cdot \mathbf{p}\right)$, respectively. Now we integrate around a loop using this propagator. In second order we look at

$$
A_{2} \equiv \frac{1}{2} \int_{-\infty}^{\infty} d t_{1} \int_{-\infty}^{\infty} d t_{2} F_{q}\left(t_{1}\right) F_{q}\left(t_{2}\right) \delta\left(t_{1}+t_{2}\right)
$$

where the $\delta$ function insures that the electron comes

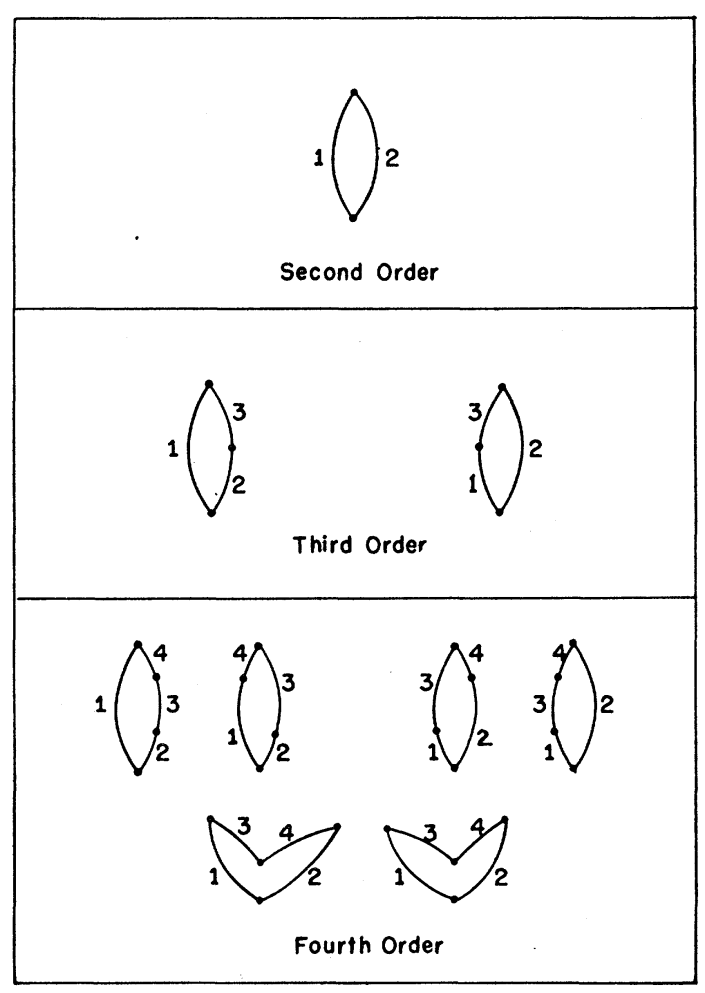

FIG. 1. The relevant second-, third-, and fourth-order processes represented diagrammatically. In second order the process may take place with or without exchange. In higher orders, exchange is neglected. 
back to its starting point. Then we have

$$
\begin{aligned}
A_{2}= & \int d^{3} p_{1} \int d^{3} p_{2} \int_{0}^{\infty} d t \\
& \quad \times \exp \left\{-\left(\frac{1}{2} q^{2}+\mathbf{q} \cdot \mathbf{p}_{1}\right) t+\left(\frac{1}{2} q^{2}+\mathbf{q} \cdot \mathbf{p}_{2}\right)(-t)\right\} \\
= & \int d^{3} p_{1} \int d^{3} p_{2} \frac{1}{q^{2}+\mathbf{q} \cdot\left(\mathbf{p}_{1}+\mathbf{p}_{2}\right)}
\end{aligned}
$$

just what we need for Eq. (8).

In third order, we look at

$$
\begin{aligned}
& A_{3} \equiv \frac{1}{3} \int_{-\infty}^{\infty} d t_{1} \int_{-\infty}^{\infty} d t_{2} \int_{-\infty}^{\infty} d t_{3} \\
& \times F_{q}\left(t_{1}\right) F_{q}\left(t_{2}\right) F_{q}\left(t_{3}\right) \delta\left(t_{1}+t_{2}+t_{3}\right) .
\end{aligned}
$$

We find

$$
\begin{array}{r}
A_{3}=2 \int d^{3} p_{1} \int d^{3} p_{2} \int d^{3} p_{3} \int_{0}^{\infty} d t_{1} \int_{-t_{1}}^{0} d t_{2} \\
\times \exp \left\{-\left(\frac{1}{2} q^{2}+\mathbf{q} \cdot \mathbf{p}_{1}\right) t_{1}+\left(\frac{1}{2} q^{2}+\mathbf{q} \cdot \mathbf{p}_{2}\right) t_{2}\right. \\
\left.+\left(\frac{1}{2} q^{2}+\mathbf{q} \cdot \mathbf{p}_{3}\right)\left(-t_{1}-t_{2}\right)\right\}=2 \int d^{3} p_{1} \int d^{3} p_{2} \int d^{3} p_{3} \\
\times \frac{1}{q^{2}+\mathbf{q} \cdot\left(\mathbf{p}_{1}+\mathbf{p}_{2}\right)} \frac{1}{q^{2}+\mathbf{q} \cdot\left(\mathbf{p}_{1}+\mathbf{p}_{3}\right)}
\end{array}
$$

which is what we need for Eq. (10).

The agreement evidently extends to all orders. It should be noted that there is a direct correspondence between the various forms of the diagram in a given order and the various time-orderings in the corresponding integral $A_{n}$.

Now let us perform the Fourier transform of the $\delta$ function in the expression for $A_{n}$. We obtain

$$
A_{n}=\frac{q}{2 \pi n} \int_{-\infty}^{\infty} d u\left[Q_{q}(u)\right]^{n},
$$

where

$$
Q_{q}(u)=\int d^{3} p \int_{-\infty}^{\infty} e^{i t u q} \exp \left\{-|t|\left[\frac{1}{2} q^{2}+\mathbf{q} \cdot \mathbf{p}\right]\right\} d t .
$$

The terms in the correlation energy contributing to the constant $C$ may then be summed, leaving aside the exchange term $\epsilon_{b}{ }^{(2)}$. We have

$$
\begin{aligned}
\epsilon^{\prime} \equiv \epsilon_{a}{ }^{(2)}+\epsilon^{(3)}+\epsilon^{(4)}+\cdots & =-\frac{3}{8 \pi^{5}} \int \frac{d^{3} q}{q^{3}} \frac{1}{2 \pi} \\
& \times \int_{-\infty}^{\infty} d u \sum_{n=2}^{\infty} \frac{(-1)^{n}}{n}\left[Q_{q}(u)\right]^{n}\left(\frac{\alpha r_{s}}{\pi^{2} q^{2}}\right)^{n-2} .
\end{aligned}
$$

This expression may now be enormously simplified when we realize that beyond the second order we are interested only in the most highly divergent part of the $q$ integral, that is, the leading term near $q=0$. We may thus take, beyond the second order, just this leading term and put the upper limit of the $q$ integral equal to some arbitrary number, which we take to be 1 .

At small values of $q$, we may approximate $Q_{q}(u)$ as follows: We apply the restrictions on $\mathbf{p}$ that $p<1$ and $|\mathbf{p}+\mathbf{q}|>1$. If $x$ is the direction cosine between $\mathbf{p}$ and $\mathbf{q}$ we see that at small $q$ the variable $x$ is restricted to the range $0 \leq x \leq 1$ and $p$ to the range $1-q x \leq p \leq 1$. Thus we have, for $q \ll 1$,

$$
\begin{aligned}
Q_{q}(u) \approx 2 \pi q \int_{0}^{1} x d x \int_{-\infty}^{\infty} d t e^{i t u q} e^{-|t| q x} \\
\quad=2 \pi \int_{0}^{1} x d x \int_{-\infty}^{\infty} d^{q} e^{i s u} e^{-|s| x}=4 \pi R(u)
\end{aligned}
$$

independent of $q$. We have put

$$
R(u)=1-u \arctan u^{-1} \text {. }
$$

We shall thus approximate $Q_{q}(u)$ by a function which is equal to $4 \pi R(u)$ when $0 \leq q \leq 1$ and which vanishes for $q>1$. In second order, we must supply a correction term that restores $\epsilon_{a}{ }^{(2)}$ to its exact value, but in higher orders the approximation is sufficient. We have, then,

$$
\begin{aligned}
& \epsilon^{\prime} \approx-\frac{12}{\pi^{3}} \int_{-\infty}^{\infty} d u \int_{0}^{1} \frac{d q}{q} \sum_{n=2}^{\infty} \frac{(-1)^{n}}{n} \\
& \times[R(u)]^{n}\left(\frac{4 \alpha r_{s}}{\pi q^{2}}\right)^{n-2}+\delta
\end{aligned}
$$

where we have put

$$
\begin{aligned}
\delta \equiv & \epsilon_{a}{ }^{(2)}-\left[-\frac{12}{\pi^{3}} \int_{-\infty}^{\infty} d u \int_{0}^{1} \frac{d q}{q}\left(\frac{1}{2} R^{2}\right)\right] \\
& =\lim _{\beta \rightarrow 0}\left\{-\frac{3}{8 \pi^{5}} \int_{\beta}^{\infty} \frac{d^{3} q}{q^{4}} \int_{\substack{p_{1}<1 \\
\left|\mathbf{p}_{1}+\mathbf{q}\right|>1}} d^{3} p_{1} \int_{\substack{p_{2}<1 \\
\left|\mathbf{p}_{2}+\mathbf{q}\right|>1}} d^{3} p_{2}\right. \\
& \left.\times \frac{1}{q^{2}+\mathbf{q} \cdot\left(\mathbf{p}_{1}+\mathbf{p}_{2}\right)}+\frac{6}{\pi^{2}} \int_{\beta}^{1} \frac{d q}{q} \int_{0}^{1} x d x \int_{0}^{1} y d y \frac{1}{x+y}\right\},
\end{aligned}
$$

which is a finite number, the logarithmic divergences cancelling.

We comment here on a difficulty which occurs in carrying out the summation over $n$. For large values of $q$, the series converges and the summation is straightforward. For small $q$, however, the series diverges, with large contributions arising from large $n$. We shall, however, assume that the result valid for large $q$ may be continued into the region of divergence. This procedure cannot be justified without a detailed investigation of the behavior of the series for large $n$; we argue, 
however, that any corrections must either vanish with large $N$ or contribute to higher powers of $\boldsymbol{r}_{s}$.

We may now perform the sum over $n$ and the integral over $q$ in Eq. (22), remarking that the integral really does cut itself off at a value of $q$ proportional to $r_{s}^{\frac{1}{2}}$, as predicted in Eq. (6). Dropping terms that vanish as $r_{s} \rightarrow 0$, we have

$$
\begin{aligned}
\epsilon^{\prime} & =\frac{3}{\pi^{3}} \int_{-\infty}^{\infty} d u[R(u)]^{2}\left[\ln \left(\frac{4 \alpha r_{s}}{\pi}\right)+\ln R(u)-\frac{1}{2}\right]+\delta \\
& =\frac{2}{\pi^{2}}(1-\ln 2)\left[\ln \left(\frac{4 \alpha r_{s}}{\pi}\right)+\langle\ln R\rangle_{\text {Av }}-\frac{1}{2}\right]+\delta
\end{aligned}
$$

where

$$
\langle\ln R\rangle_{\mathrm{Av}}=\int_{-\infty}^{\infty} d u R^{2} \ln R / \int_{-\infty}^{\infty} d u R^{2} .
$$

We see that Eq. (24) confirms our value of $\left(2 / \pi^{2}\right)$ $\times(1-\ln 2)$ for the constant $A$. For the constant $C$, we have

$$
C=\frac{2}{\pi^{2}}(1-\ln 2)\left\{\ln \left[\frac{4}{\pi}\left(\frac{4}{9 \pi}\right)^{\frac{1}{3}}\right]-\frac{1}{2}+\langle\ln R\rangle_{\mathrm{Av}}\right\}+\delta .
$$

Now Pines $^{3}$ has found the value -0.0508 for $\delta$, and numerical integration yields the value -0.551 for $\langle\ln R\rangle_{\mathrm{Av}}$. The multiple integral (9) for $\epsilon_{b}{ }^{(2)}$ has been evaluated by the Monte Carlo method, with the result $\epsilon_{b}{ }^{(2)}=0.046 \pm 0.002$. Substituting these numbers into (26), we find

$$
\begin{aligned}
& C=-0.096 \pm 0.002, \\
& \epsilon_{c}=0.0622 \ln r_{s}-0.096+O\left(r_{s}\right) .
\end{aligned}
$$

The expression given by $\mathrm{Pines}^{3}$ is $0.0311 \ln r_{s}-0.114$ $+O\left(r_{s}\right)$, although if the correction mentioned in footnote 4 is taken into account one gets by Pines' method the result $0.0622 \ln r_{s}-0.158+O\left(r_{s}\right)$.

In the Appendix we evaluate $\epsilon_{c}$ by Macke's method and obtain $0.0622 \ln r_{s}-0.128+O\left(r_{s}\right)$.

We see that the approximations of Macke and Pines tend to overestimate the magnitude of the constant term $C$ in the correlation energy.

In conclusion, let us discuss the calculation of the next correlation to $\epsilon$. In order to include all terms that are genuinely of order $r_{s}$ or $r_{s} \ln r_{s}$, we must improve the present calculation in three ways;

(i) The contribution to $\epsilon^{\prime}$ from Eq. (19) must be treated more carefully than in Eq. (22), so that terms of order $r_{s}$ are retained.

(ii) We must calculate the contribution from the diagrams in Fig. 1 beyond the second order when one exchange is permitted in each process. Beyond the third order we may employ the crudest approximation that preserves the leading divergence. (iii) The remaining third-order processes are the following:

$1+2 \rightarrow 1^{\prime}+2^{\prime}$

$\left.1^{\prime}+2^{\prime} \rightarrow 1^{\prime \prime}+2^{\prime \prime}\right\}$ rescattering.

$1^{\prime \prime}+2^{\prime \prime} \rightarrow 1+2$

$\left.\begin{array}{l}1+2 \rightarrow 1^{\prime}+2^{\prime} \\ 1^{\prime}+3 \rightarrow 1^{\prime}+3\end{array}\right\}$ direct and exchange scattering with $\left.\begin{array}{l}1^{\prime}+3 \rightarrow 1^{\prime}+3 \\ 1^{\prime}+2^{\prime} \rightarrow 1+2\end{array}\right\}$ unexcited particles.

Those are both logarithmically divergent and must be combined with a sequence of terms similar to those summed to remove the second-order divergence. The methods presented above are easily generalized to this case.

The authors would like to thank Dr. Richard Latter for many valuable discussions and Mr. J. I. Marcum and Mr. H. Kahn for the Monte Carlo computation of $\epsilon_{b}^{(2)}$.

\section{APPENDIX. APPROXIMATION OF MACKE}

The method of $\mathrm{Macke}^{2}$ is suggested by the earlier work of Wigner. ${ }^{1}$ It consists of summing, instead of the complete set of diagrams indicated in Fig. 1, just the first diagram in each order. Under the integral sign these form a simple geometric series. We obtain, in place of Eq. (19), the following:

$$
\begin{aligned}
\epsilon^{\prime} & \approx \frac{3}{8 \pi^{5}} \int \frac{d^{3} q}{q^{4}} \int_{\substack{p_{1}<1 \\
\left|\mathbf{p}_{1}+q\right|>1}} d^{3} p_{1} \sum_{n=1}^{\infty}(-1)^{n} \\
& \times\left(\int_{\substack{p_{2}<1 \\
\left|\mathbf{p}_{2}+\mathbf{q}\right|>1}} d^{3} p_{2} \frac{1}{q^{2}+\mathbf{q} \cdot\left(\mathbf{p}_{1}+\mathbf{p}_{2}\right)}\right)^{n}\left(\frac{\alpha r_{s}}{\pi^{2} q^{2}}\right)^{n-1} .
\end{aligned}
$$

Making an approximation analogous to (20) in the orders beyond the second, we find instead of (22) the expression

$$
\begin{aligned}
\epsilon^{\prime} \approx \delta+\frac{6}{\pi^{2}} \int_{0}^{1} \frac{d q}{q} & \int_{0}^{1} x d x \sum_{n=1}^{\infty}(-1)^{n} \\
& \times\left(\int_{0}^{1} \frac{y d y}{x+y}\right)^{n}\left(\frac{2 \alpha r_{s}}{\pi q^{2}}\right)^{n-1} .
\end{aligned}
$$

We may now, as before, perform the sum over $n$ and the integral over $q$, dropping terms that vanish as $r_{s} \rightarrow 0$. Putting $I=\int_{0}^{1} y d y /(x+y)$, we find

$$
\epsilon^{\prime} \approx \delta+\frac{3}{\pi^{2}} \int_{0}^{1} x d x I \ln \left(\frac{2 \alpha r_{s} I}{\pi}\right)
$$

The approximate value of $C$ is then

$$
\begin{aligned}
C & \approx \epsilon_{b}{ }^{(2)}+\delta+\frac{3}{\pi^{2}} \int_{0}^{1} x d x I \ln \left(\frac{2 \alpha I}{\pi}\right) \\
& \approx \epsilon_{b}{ }^{(2)}-0.174 .
\end{aligned}
$$

